Published online 2017 April 13.

Abstract

\title{
Test-Retest Reproducibility and Robustness Analysis of Recurrent Glioblastoma MRI Radiomics Texture Features
}

\author{
Isaac Shiri, ${ }^{1,}$ Hamid Abdollahi, ${ }^{2}$ Sajad Shaysteh, ${ }^{2}$ and Seied Rabi Mahdavi ${ }^{1}$ \\ ${ }^{1}$ Department of Medical Physics, Iran University of Medical science, Tehran, Iran \\ ${ }^{2}$ Iran University of Medical Science, Tehran, Iran \\ "Corresponding author: Isaac Shiri. E-mail: isaac.sh92@gmail.com
}

Received 2016 December 21; Accepted 2017 February 08.

\begin{abstract}
Background: Advanced quantitative information such as radiomics features derived from magnetic resonance (MR) image may be useful for outcome prediction, prognostic models or response biomarkers in Glioblastoma (GBM), knowledge about their Reproducibility and robustness is essential. The purpose of this study was to investigate the reproducible and non-redundant radiomics texture features in oncological GBM MRI based on test-retest setup.

Methods: Thirteen patients with recurrent GBM who underwent repeated MR imaging approximately two day interval were subjected to this study. Lesions were segmented using competitive region-growing based algorithm. Following delineation and segmentation of lesions, 158 quantitative 3D features based on intensity histograms (IH), gray level run-length (GLRLM), gray level cooccurrence (GLCM), gray level size-zone texture matrices (GLSZM), neighborhood-difference matrices (NDM), and geometric features were extracted from the 3D-tumor volumes of each lesion. For every radiomics feature, test-retest was assessed with the intra-class correlation coefficient (ICC) and the concordance correlation coefficient (CCC) and finally the most reproducible and robust radiomics features were selected.

Results: Results shows that the '74\%' of assessed radiomics features had a high test-retest stability in terms of their ICC. There were 107 radiomics features with 'CCC $>0.95$ ' and 49 radiomics features were non-redundant after grouping features with 'R2Bet $\geq 0.95$ '. Conclusions: Test-retest and correlation analyses have identified non-redundant radiomics features and this feature are prone to errors if they employed as quantitative biomarker for GBM image analysis. However when we use robust and redundant feature, quantitative image radiomics features are informative and prognostic biomarkers for GBM Magnetic resonance imaging.
\end{abstract}

Keywords: Radiomics, GBM, MRI, Feature

This is an abstract presented in the 33rd Iranian congress of radiology (ICR) and the 15th congress of Iranian radiographic science association (IRSA). 